\title{
APOLOGIES IN L1 AND LINGUA FRANCA ENGLISH: A CONTRASTIVE PRAGMATIC STUDY OF TRIPADVISOR RESPONSES
}

\author{
Christopher Hopkinson
}

\begin{abstract}
The article reports on a study of restaurant-owners' public online responses to negative customer reviews on the TripAdvisor website, exploring the differences between the practices used by L1 English and lingua franca English (ELF) speakers when performing public apologies. The focus is on the occurrence and linguistic realizations of two key components of apologies: illocutionary force indicating devices (IFIDs) and accounts of the incident. The results indicate certain differences between the L1 and ELF responses both in the use of IFIDs (the IFIDs in the ELF responses are more frequently ambiguous in terms of their illocution) and in the amount of facework done (the L1 responses use facework resources more proficiently, while the ELF responses are more face-neutral).
\end{abstract}

\section{Keywords}

apologies, contrastive pragmatics, facework, internet genres, lingua franca, speech acts

\section{Introduction}

English as a lingua franca (ELF) has emerged as a major field of linguistic research in recent years. Empirical studies have produced descriptive accounts of ELF on various levels, ranging from phonology through lexicogrammar to pragmatics, and extensive ELF corpora continue to be compiled (e.g. the Helsinki-based ELFA corpus of academic ELF and the Vienna-Oxford VOICE corpus). In this study I seek to contribute to the growing body of work exploring the pragmatic aspects of ELF - a line of research that can be traced back to Meierkord's pioneering work published two decades ago (Meierkord 1996). Recent empirical studies of ELF pragmatics have addressed a broad range of issues, including the use of stance markers and other discourse markers to express intersubjectivity (House 2013, Pullin 2013), face preservation strategies used in ELF interaction (Walkinshaw and Kirkpatrick 2014), disagreement among ELF speakers (Maíz-Arévalo 2014), and vagueness (Metsä-Ketelä 2016); a synthetic overview of the development of ELF pragmatic research is given in Kecskes (2013).

In this study I explore the occurrence and linguistic realizations of one particular speech act - apologies - in restaurant-owners' public responses to 
negative customer reviews posted on the TripAdvisor website. This recently emerged genre represents a rich source of naturally occurring data offering insights into a range of pragmatic practices. The present study takes a contrastive approach, with a corpus consisting of two equal halves - an ELF component and an L1 component. It thus belongs within a long-established line of contrastive pragmatic research that seeks to compare various speech acts and their realizations in different language communities. Speech act-based contrastive studies have primarily focused on the practices of L1 users (including gender-differentiated practices among speakers of the same language), though some research has compared the practices of L1 and L2 speakers (e.g. Lee 2016, investigating refusal styles). Speech acts explored in contrastive studies include challenges (Fetzer 2011), compliments and compliment responses (e.g. Holmes 1988, LorenzoDus 2001, Válková 2012), refusals (Lee 2016), and requests (Blum-Kulka et al. 1989, Ogiermann 2009b). The speech act I investigate here - apologies - has received quite extensive coverage in contrastive pragmatics since Blum-Kulka et al. (1989), which represented a milestone in the field. Some work has contrasted apology practices in different languages and cultures (e.g. Suszczyńska 1999, Márquez Reiter 2000), while other studies have compared apologies performed in the same language by L1 and L2 speakers (e.g. Trosborg 1995, Bella 2014); for an overview of contrastive studies cf. e.g. Ogiermann (2009a: 61ff.).

The research presented here differs from most previous contrastive pragmatic work on apologies in two main ways. Firstly, most research on apologies (both contrastive and non-contrastive) has focused on apologies in the private domain; as Page observes in her study of online responses to customer complaints on Twitter, "linguistic studies which examine apologies made in public contexts are in their infancy" (Page 2014: 32). The distinction between apologies in the private and public domains is not a trivial one; public apologies acquire an extra layer of pragmatic complexity because the speaker has to take into account not only the face concerns of the person(s) to whom the apology is directly addressed, but also the presence of third parties who are observing the apology. This presence of an audience lends public apologies a strong persuasive dimension, and some studies of such apologies (e.g. Benoit 1995, Kampf 2009) have explored their role as a means of image repair in crisis communication. Secondly, most research on public apologies has focused on prominent, high-profile examples of apology discourse from domains such as domestic or international politics (e.g. Harris et al. 2006, Kampf 2009) or the news (Gruber 2011), whereas the present study explores apologies made in a more everyday context, as part of responses to customer complaints. Such low-key public apologies still remain somewhat under-researched - though, as the ongoing social development of social media 
continues to bring formerly private genres (such as customer complaints and responses) more and more into the public domain, we can expect this type of apology to become an increasingly common feature of public discourse in the coming years.

\section{The corpus}

The corpus analyzed for this study consists of 1,000 responses to negative reviews of restaurants, cafés, bars and pubs posted on TripAdvisor. (The word 'restaurant' is used in this text as an umbrella term denoting all these types of establishments.) It comprises two identically sized subcorpora: 500 responses representing L1 English and 500 representing lingua franca English (ELF). The L1 responses were posted by restaurants in Manchester (United Kingdom), while the ELF responses were from restaurants in Prague (Czech Republic).

The corpus was compiled between March and August 2016 using the following process. TripAdvisor uses a rating system whereby reviewers (customers) grade establishments on a scale from a minimum 1 point ("terrible") to a maximum 5 points ("excellent"). For the purposes of this study, negative reviews were defined as those rating the establishment as 1 or 2 points ("terrible" or "poor", respectively). If the establishment-owner posted a response to a negative review, the response was included in the corpus - with the exception of hostile, aggressive responses, which were omitted (being of little relevance to a study of apologies). The responses were collected from establishments listed on TripAdvisor in alphabetical order, starting from A and proceeding through the alphabet until 500 responses had been amassed. The number of responses collected from each establishment was limited to 15 in order to ensure that highly prolific respondents were not overly dominant, which would have made the data less representative. The same principle was applied to establishments which posted highly standardized responses, varying only in minor details such as the name of the complainant in the opening part of the response; in order to avoid large numbers of such responses distorting the data, only one example of each standardized response was included in the corpus. The responses cover approximately a five-year period. The structure of the corpus is summarized in Table 1. 


\begin{tabular}{|l|l|l|}
\hline \multirow{2}{*}{} & \multicolumn{2}{|c|}{ Subcorpora } \\
\cline { 2 - 3 } & Manchester (L1) & Prague (ELF) \\
\hline Total No. of responses & 500 & 500 \\
\hline Total No. of establishments & 120 & 146 \\
\hline Total No. of words (approx.) & 65,000 & 63,000 \\
\hline Average words per response & 130 & 126 \\
\hline Period covered by responses & October 2011 - July 2016 & March 2011 - July 2016 \\
\hline
\end{tabular}

Table 1: Corpus structure

The corpus was subjected to a manual qualitative analysis to identify the apology strategies used by the respondents and their means of realization. In the next phase of the analysis, simple descriptive quantitative data on the occurrence of these strategies and realizations was collected in order to enable a comparison of the L1 and ELF subcorpora. Although the data is entirely in the public domain (reviewers and respondents contribute to TripAdvisor in the full knowledge that their words will potentially be read by large numbers of people), all cited examples have been anonymized where necessary to conceal the identity of the parties.

Two caveats should be made at this point regarding the research design. Firstly, it is of course possible that some of the Manchester responses were written by non-native speakers of English (and vice versa for the Prague responses). However, there is no way of reliably identifying the native/non-native status of a respondent on TripAdvisor, and for the purposes of this study it was assumed that such cases would not be numerous enough to cause significant distortion of the data. (In any case, many non-native speakers of English who are longterm residents in the UK or other English-speaking countries will, over time, increasingly develop native-like competence and patterns of language use, rendering the distinction between native and non-native speakers a somewhat problematic one.) This study should not therefore be seen as attempting to draw a simplistically clear-cut distinction between L1 and ELF speakers, but rather as exploring the different ways in which English is used in two distinctly different environments.

Secondly, it is undoubtedly true that observable differences in linguistic choices may result not only from differences in speaker status (L1 vs. ELF), but also from other factors - particularly cultural differences (depending on 
individual speakers' backgrounds). However, this study consciously does not attempt to seek cultural explanations for its data. The reason for this is that the ethnically diverse nature of the restaurant industry would make any such attempts practically unworkable. The Prague responses did not originate in a Czech monoculture; the respondents include not only Czechs, but also representatives of numerous other cultures (French, Greek, Indian, Italian, Japanese, Mexican, Russian, etc.), so tracing the influence of respondents' cultural background would not be a viable undertaking, at least with a corpus of this size. For the same reason this study does not attempt to seek explanations for its data in possible linguistic interference from respondents' L1.

\section{Online complaint responses as a genre}

Theories of genre rooted in a systemic-functional framework (e.g. Martin 1997, Martin \& Rose 2008) view genres as "staged, goal-oriented social processes" (Martin 1997: 13). Martin's 'stages' are analogous to what Swales (1990) and Bhatia $(1993$, 2004) term 'moves' - the sequentially arranged structural elements of a genre, each with its own distinct rhetorical purpose. It is not the aim of this study to explore the move structures found in the responses, and this aspect of the genre will only be mentioned in passing. More relevant are the other two components of Martin's definition - the concept of genres as social processes, and their goal-oriented nature.

\subsection{The genre as a social process}

With regard to genres as social processes, two key aspects of public complaint responses are of particular relevance to the apology practices explored in this study: the participatory framework of the discourse in which the genre is embedded, and the roles and relative status of the participants.

Before the emergence of websites such as TripAdvisor, customer complaints were typically dealt with in private correspondence between the complainant and a representative of the business; this involves a simple dyadic participatory framework. In public complaint responses the participatory framework is more complex; it also includes readers visiting the site, who constitute an audience of 'side participants' (Haugh 2013, Kádár \& Haugh 2013). I term these side participants 'third parties'; a third party is "a ratified listener to whom an utterance is not addressed but who is fully entitled to listen to it and make inferences, according to the speaker's communicative intention" (Dynel 2014: 40).

The second key aspect of the genre as a social process is the relative status of the participants. The default social situation in which public complaint responses are embedded is based on a clear imbalance of status (power) between 
the respondent (i.e. the speaker - S) and the complainant (i.e. the hearer $\mathrm{H})$. The act of paying for a service establishes a relationship based on mutual rights and obligations; within the bounds of the commercial relationship, the customer essentially buys power over the service provider. This in turn creates a social expectation that the customer will be treated with a certain degree of deference (though the degree of deference that is felt to be appropriate will differ between cultures, and it will also be dependent on factors such as the type and price-bracket of the establishment). Although respondents sometimes violate this expectation of deference (by criticizing the review as unfair, malicious or dishonest), such cases are marginal in terms of quantity, and responses featuring this type of behaviour were omitted from the corpus as they are of little relevance to a study of apologies.

\subsection{The genre as a goal-oriented process}

The other key component of Martin's definition of genre (Martin 1997: 13) with implications for the topic of this study is the "goal-oriented" nature of genres. Public complaint responses typically pursue two main communicative goals. Firstly, they offer respondents a chance to offer explanations, remedy or compensation, and generally to show that they are sympathetic and responsive to their customers' needs - all helping to repair their damaged image. The genre thus represents a powerful marketing tool. A crucial factor here is the public nature of the discourse: respondents are attempting not only to persuade and win over the original complainant, but also to create a good impression on thirdparty readers. Most of these third parties are likely to be potential diners using the site to help them choose a restaurant, so a well-handled response ultimately offers the possibility of significant commercial gain (or at least the avoidance of significant loss).

The second main communicative goal of this genre does not involve the business's image as such, but rather the mutual relationship between the complainant and the respondent. Most public complaint responses centre around some form of apology (cf. Page 2014). One of the primary purposes of apologizing is the restoration of social (relational) equilibrium between the parties (Holmes 1990, Davies et al. 2007). Equilibrium does not necessarily mean equality of status or power; it involves the maintenance of a stable relationship between parties, based on mutual and/or social expectations - even if these expectations mean, as is the case here, that the parties' status is unequal. The circumstances motivating the complaint - the business's failure to meet some of its obligations to the customer - represent a disturbance of this equilibrium, so apologies offer an opportunity to restore it. 


\section{The speech act of apology}

A highly influential study of apologies that laid the foundations for much future research was Blum-Kulka et al. (1989), which identified five major strategies that may be used in apologies:

1. 'illocutionary force indicating device' (IFID) - IFIDs are "formulaic, routinized expressions in which the speaker's apology is made explicit" (Blum-Kulka et al. 1989: 290), e.g. I apologize or I am sorry;

2. taking on responsibility;

3. explanation or account (i.e. an account of the incident that caused offence);

4. offer of repair;

5. promise of forbearance, e.g. It won't happen again.

(Blum-Kulka et al. 1989: 289)

Subsequent research has proved this framework to be quite robust, and the corpus analyzed for the present study likewise confirms its general validity.

Due to space constraints, this study omits items 4 and 5 in the above list (which in my data tend to occur as separate moves, towards the end of the response) and instead focuses solely on two main components of apologies: 1. the IFID, and 2. the speaker's account of the incident that provoked the negative review. I view the 'account' in a broad sense; this component of the apology typically incorporates not only the speaker's (S's) re-narration of the incident, but also two more elements that are woven into the fabric of the narrative: S's explicit negative evaluation of the restaurant's performance (e.g. You clearly received appalling service from us.) and S's acceptance of the restaurant's responsibility or culpability for what happened (e.g. We hold our hands up - no excuses, we got it wrong.). What I term the 'account' thus subsumes two of the strategies listed by Blum-Kulka et al. (items 2 and 3 in the list cited above).

Ogiermann (2009a: 137 ff.), drawing on work by Bergman and Kasper (1993), notes that accounts may be either downgraded (if S downplays the severity of the offence or avoids taking full responsibility for it) or upgraded (if $\mathrm{S}$ accepts the severity of the offence and foregrounds their own culpability). These are core facework strategies in apologies. An apology represents a facethreatening act (FTA) to the speaker, so downgrading the account represents an attempt to mitigate this face threat and save S's face. By contrast, upgrading an account involves a somewhat more complex facework mechanism: although S's explicit negative self-evaluation damages one aspect of their face (i.e. the part of S's face that concerns their competence as a provider of services to the public), it simultaneously asserts a different aspect of S's face, foregrounding 
their ability to accept criticism, their respect for customers' views, and their general "good listenership" - in other words, various positive moral qualities. $\mathrm{S}$ thus strategically sacrifices 'competence face' in order to enhance 'morality face', in a mechanism that I term 'face compartmentalization'.

The aim of this study is to compare the apologies performed by L1 and ELF speakers with a focus on the two key components of the apology discussed aboveIFIDs and accounts. The analysis explores both the occurrence (frequency) and linguistic realizations of apologies in order to answer two main questions:

1. Does the data reveal differing preferences between L1 and ELF speakers in terms of the strategies used in their apologies?

2. Does the data reveal differences in the typical linguistic realizations of apologies between L1 and ELF speakers?

\section{Results and discussion}

This section is divided into two parts, each addressing one of the two main aspects of apologies covered in this study: IFIDs and accounts.

\subsection{Illocutionary force indicating devices (IFIDs)}

IFIDs are the canonical resources for the realization of speech acts. IFIDs of apology are defined by Blum-Kulka et al. (1989: 290) as "formulaic, routinized expressions in which the speaker's apology is made explicit". Blum-Kulka et al. (ibid.: 290) and Ogiermann (2009a: 95 ff.) list a variety of apology IFIDs, including I apologize, my apologies, I'm sorry for ..., I regret ..., excuse me, forgive me, etc.

The variety of IFIDs in my corpus is highly restricted, with the repertoire consisting essentially of two resources: 1) the performative verb apologize, or structures incorporating the nominal version apology/apologies; 2) structures incorporating sorry. The illocutionary force of these acts may be upgraded (boosted) by adding a variety of lexical means, typically adverbial or adjectival forms (e.g. I apologise unreservedly, I can only apologise profusely, You have my sincere apologies, I am so sorry, We are deeply sorry). The apology may also be upgraded by framing it as a polite request directed at the hearer (e.g. Please allow me to apologise to you, please accept my apologies) or by attaching it to another act which indexes the relational inequality between $\mathrm{S}$ and $\mathrm{H}$, positioning $\mathrm{S}$ in the role of the supplicant and granting $\mathrm{H}$ the right to either accept or reject the apology (I hope you can accept my apology).

While the verb apologize and the nominal version apology/apologies are unambiguous in terms of their illocution, the use of structures involving sorry is more problematic. The form sorry is polyfunctional; essentially it indexes the 
speaker's affective stance (sympathy, regret), but it can also be used to perform apologies. This dual function of sorry was recognized by Austin, who classed the formula I'm sorry both as one of "numerous conventional expressions of feeling" (Austin 1975: 80-81) and as a primary performative for apologies (ibid.: 79,83 ). In cases where sorry is used to perform an apology, typical patterns of complementation include e.g. I am sorry that we failed to meet our usual standards, We are sorry to have let you down, I am so sorry for the poor quality of service you received. The speaker is typically encoded as the agent in the complement clause, or if agentless structures are used (as in the third of the examples listed above) the incident is evaluated in such a way that it leaves no doubt as to the speaker's culpability. In such cases, the use of sorry clearly fulfils one of the essential felicity conditions for a genuine apology - the speaker's remorse for what happened (i.e. not just S's regret that something happened, but also S's acceptance of responsibility; see e.g. Jucker \& Taavitsainen 2008: 230).

Crucially, however, the expression of S's affective stance through the form sorry does not necessarily mean that an apology is being performed. The corpus provides numerous examples of cases in which the complementation of sorry does not encode $\mathrm{S}$ as the responsible agent (even implicitly), but instead focuses on $\mathrm{H}$ as the patient and/or experiencer, e.g. I was very sorry to hear about your experience, I am sorry to read that you did not enjoy your meal. Clearly such an act, on its own, does not constitute an apology; although S expresses sympathy with H's experience, there is no acceptance of culpability. The illocution of such acts is closer to that of a condolence (I am very sorry about your loss).

In some cases the illocution of sorry is not made unambiguously clear by the utterance itself; it is only the wider context (typically the preceding and following utterances) which provides the necessary interpretative cues. For example:

I am very sorry about your experience. The mistakes that were made should not have happened.

Here the utterance highlighted in bold type, if viewed in isolation, is pragmatically ambiguous - it could potentially be interpreted as an apology, or it could be solely an expression of affective stance (sympathy). However, this ambiguity is resolved by the utterance that follows it, which clearly implies an acceptance of responsibility - a typical element of an apology. Likewise, in the following example -

(2) We endeavour to hire friendly and helpful staff that are employed both as a security measure but also as a door host to assist customers on their entrance to [restaurant name]. We're sorry that this damaged your experience of [restaurant name] but we do feel it is necessary and helpful. 
- the illocutionary ambiguity of the speech act marked in bold type is resolved by both the preceding and following utterances, which make it clear that this is in no way an apology.

However, this ambiguity is not always resolved, and some utterances in the corpus lack illocutionary clarity: sorry could plausibly be interpreted as merely expressing S's affective stance (sympathy, regret), but it could also, equally plausibly, be interpreted as implying S's acceptance of responsibility, and thus performing an apology. This typically occurs in brief, perfunctory responses which fail to provide adequate contextual support for either interpretation. The utterance thus exists in a kind of pragmatic limbo, with the text allowing for both possible interpretations. In such cases, $\mathrm{H}$ is typically encoded as the experiencer, and S expresses sympathy with H's (subjective) experience. For example:

I am very sorry that you did not thoroughly enjoy your dining experience during your last visit.

\section{I am sorry you didn't feel comfortable in our restaurant.}

In the absence of any interpretative cues from the wider context, it is quite plausible to interpret such utterances as cases of S implicitly accepting some degree of responsibility for H's experience - or, at the very least, such an interpretation cannot be rejected out of hand. The illocutionary ambiguity thus remains unresolved.

In view of the issues outlined in the preceding paragraphs, the quantity data presented below splits the occurrences of the IFID sorry into two categories, distinguishing between cases in which the illocution is clearly that of an apology and cases in which the illocution is not clear. Figure 1 gives a graphic depiction of the total number of occurrences of apology IFIDs in the corpus (including those occurrences that may plausibly be interpreted as apologies, but are not unambiguously identifiable as such). The numerical data forming the basis for the graphic representation is given in table form in the Appendix. 


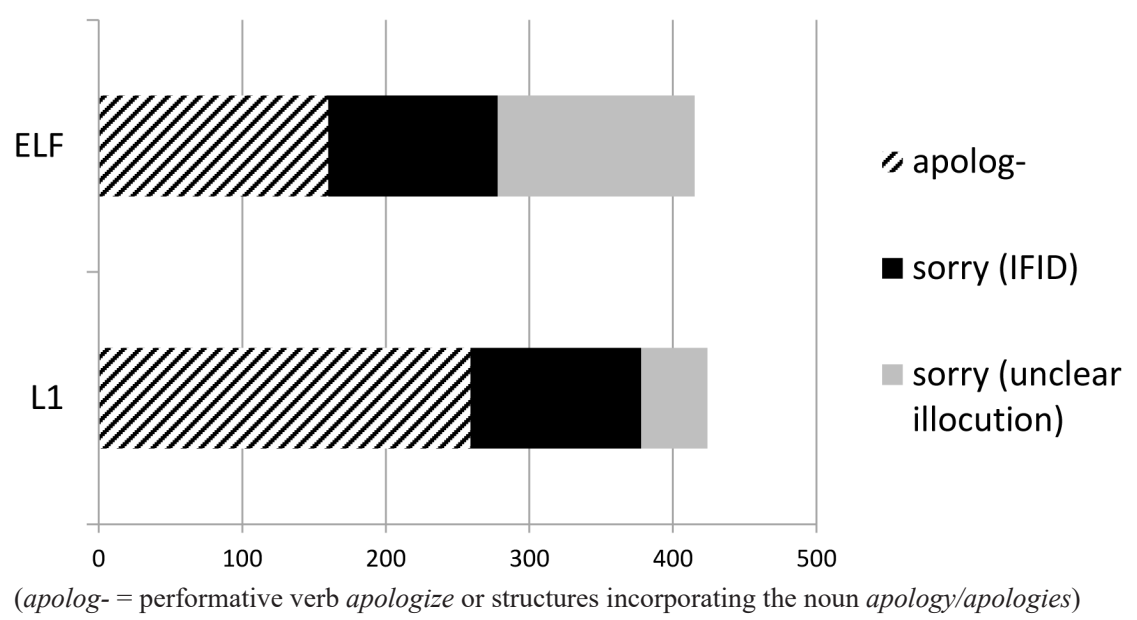

Figure 1: IFIDs of apology (No. of occurrences)

The overall quantity of apology acts (including those acts with unclear illocution that could plausibly be interpreted as apologies) is very similar in both the L1 (Manchester) and ELF (Prague) data; this is to be expected, as the communicative purpose of the genre is the same in both locations.

However, the data does reveal two main differences between L1 and ELF realizations of the IFID. Firstly, the L1 responses show a distinct preference for the performative verb apologize or the related nominal version apology/ apologies (which are clearly predominant over sorry), while the ELF responses show a slight preference for sorry. The L1 preference for apolog- forms in my data is strongly at variance with the results reported by Ogiermann (1999a), who found that I'm sorry or sorry accounted for the overwhelming majority (98\%) of the apology IFIDs used by her British respondents (ibid.: 95-96). The most likely explanation for this discrepancy is the markedly different nature of the data. Ogiermann's study used a questionnaire to collect data from her subjects, and the scenarios in the questionnaire were designed to elicit immediate apologies given by the subject to the offended party in a situation of face-toface oral communication; in most of the scenarios the subjects and the offended party are friends or at least acquaintances. My data, on the other hand, involves written, non-face-to-face communication between parties who are not personally acquainted. An explanation may therefore be that the performative verb $I$ apologize, and structures incorporating related nominal forms (Please accept my apologies), are not generally felt to be appropriate in the relatively informal 
contexts that characterize Ogiermann's data, where the somewhat more casual sorry is a more natural choice.

The second noticeable difference between the L1 and ELF data is that the occurrence of sorry with ambiguous illocution is markedly more frequent among the ELF speakers. Two possible explanations present themselves: either the speakers are deliberately using sorry with an ambiguous illocution for some kind of strategic purpose, or they are simply unaware of - or unconcerned by - the lack of illocutionary clarity in their text.

With regard to the possible deliberate use of illocutionary ambiguity, it has been noted by several researchers that the polyfunctional, pragmatically 'slippery' nature of sorry makes it an ideal resource for performing what can be termed 'non-apologies'. Kampf (2009) uses this term to denote rhetorical constructs which enable speakers to (superficially) appear to be offering an apology while in fact minimizing their own responsibility in an attempt to save face. Linguistic realizations of non-apologies include "using a verb with several pragmatic functions that does not necessarily count as an apology" (Kampf 2009: 2261; here Kampf is referring to a Hebrew verb that is functionally equivalent to the English adjective sorry). Similarly, Gruber (2011) identifies the polyfunctionality of I'm sorry as a feature of what she terms 'pseudo-apologies' - a "deviant type" of apology that indexes a stance lacking in remorse (Gruber 2011: 93). The point of non-apologies, therefore, is merely to "say sorry" as a surface realization, in the hope of placating the hearer, but without the acceptance of culpability that is an essential prerequisite for a genuine apology.

One possible explanation for the higher frequency of sorry with ambiguous illocution among the ELF speakers could therefore be that they are deliberately taking advantage of the polyfunctional nature of sorry in order to perform nonapologies. However, such an explanation is perhaps somewhat counterintuitive, if we take into account that this type of linguistic behaviour requires a certain degree of pragmatic proficiency which would be more likely to be possessed by L1 speakers. The other possibility - which, I would argue, is considerably more plausible as an explanation - is that the ELF speakers are in fact constrained by their relatively limited linguistic and pragmatic competence, which sometimes causes them to unwittingly produce utterances with ambiguous illocution even though their actual intention may be either to apologize or solely to express affective stance (sympathy, regret).

The quantity data on the functions of sorry in the corpus offers an additional insight into the differing use of sorry by L1 and ELF speakers. All occurrences of sorry in the corpus were analyzed to determine their pragmatic function (apology, solely affective stance, or ambiguous illocution), and a graphic depiction of the data is given in Figure 2: 


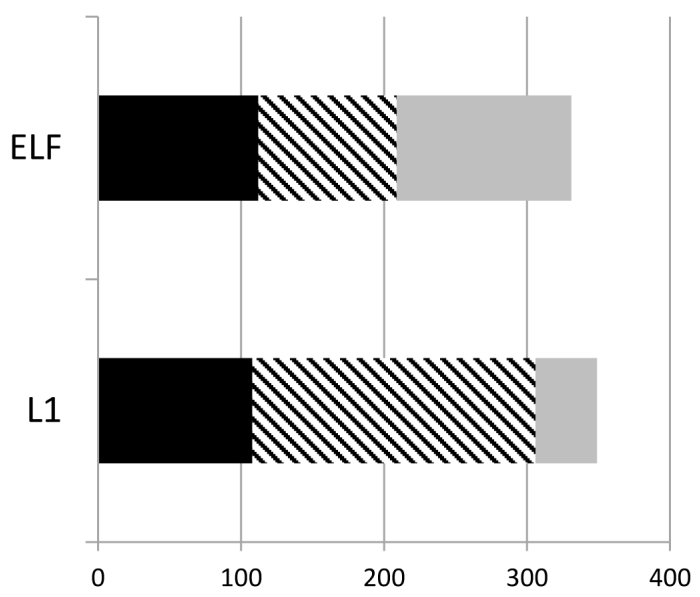

- apology IFID

$\aleph$ affective stance

ambiguous illocution

Figure 2: Functions of sorry (all occurrences of sorry in the corpus with a particular function)

The overall frequency of sorry does not differ markedly between ELF and L1 speakers, but ambiguous illocutions are considerably more numerous in the ELF responses. The ELF responses also contain considerably fewer occurrences of unambiguous sorry expressing solely affective stance (sympathy, regret). This echoes the data presented in Figure 1 showing that the ELF responses contain fewer occurrences of unambiguous apology IFIDs; put simply, unambiguously clear illocution - whether of apology or solely of affective stance - is considerably more common in the L1 responses than in their ELF counterparts.

If we accept the explanation suggested above - that ELF speakers are constrained by their relatively limited linguistic and pragmatic competence, which sometimes causes them to unwittingly produce utterances with ambiguous illocution - then this raises an interesting question of how such behaviour should be viewed. One view would be that the pragmatic ambiguity of sorry in ELF is an example of the "pragmatic failure" (Leech 2014: 262) that is typically associated with speakers of a foreign language - manifested here in their reduced ability to produce utterances with adequate illocutionary clarity. This view sees ELF as a form of 'learner English', which, when measured against the standards of native speaker (L1) language use, is to some extent defective. However, an alternative view sees ELF as a functional variety of English in its own right, operating on its own terms - which are not necessarily the same terms used by L1 varieties. This view (exemplified in the work of leading ELF researchers such as Mauranen and Seidlhofer) is based on "the idea that the international use of English does 
not have to be anchored to the standards set by inner circle countries such as the United States and the UK" (Leech 2014: 261).

Taking this alternative view, we can observe that sorry in fact performs a somewhat different function in ELF than it does in L1 English. In ELF it is used more as a formulaic 'chunk' (cf. e.g. Mauranen 2009), i.e. a conventionalized unit that forms part of a speaker's repertoire and is used for purposes of managing interaction in specific situations - in this case, the situation in which $\mathrm{S}$ needs to perform some kind of public apology. Although both native and non-native speakers use chunks in this way, research has suggested that this may be more common practice in ELF than in L1 English; Carey (2013) found that many conventional chunks were more frequent in an ELF corpus than a comparable L1 corpus. It may therefore be the case that ELF speakers in fact use structures with sorry as a pre-packaged, ready-made chunk which can be deployed almost universally to express S's stance whenever writing an apology, and that the primary purpose of this chunk is not to convey any precise desired illocution, but rather to facilitate the writing process. Such a chunk is thus analogous to Toury's (1995: 267-268) concept of the 'repertoreme' in translation studies - a conventionalized translation solution that forms part of an individual translator's repertoire, whose purpose is to save time and effort by functioning as a readymade option whenever the translator encounters a particular situation (i.e. the need to translate a particular expression or to render a particular source language structure in the target language). ELF speakers using structures with sorry thus may not only be unaware of the lack of illocutionary clarity in their text; they may indeed be largely unconcerned by it. Sorry is chosen primarily for its convenience (it is easy to use) and because it is felt to be appropriate to the given situation (needing to write an apology). This does not mean that ELF speakers are experiencing pragmatic failure, but rather that they are using a particular linguistic resource in a slightly different way than L1 speakers.

\subsection{Accounts (downgrading and upgrading)}

Besides IFIDs, the other main aspect of apologies covered in this study is S's account of the original incident that provoked the negative review (BlumKulka et al. 1989: 289). These accounts range from lengthy re-narrations of the incident (relatively infrequent in the corpus) to very brief characterizations of the incident, such as your distressing experience or the poor service you received (much more typical in the data). As has been mentioned above (in Section 4), two distinct elements are frequently woven into the fabric of the account: S's explicit negative evaluation of the restaurant's performance (e.g. You clearly received appalling service from us) and S's acceptance of the restaurant's responsibility 
or culpability for what happened (e.g. We hold our hands up - no excuses, we got it wrong).

The force of this component of the apology may either be downgraded or upgraded. Downgrading occurs if $\mathrm{S}$ downplays the severity of the offence or avoids taking full responsibility for it; this is an attempt to mitigate the face threat to S. Upgrading occurs if S accepts or plays up the severity of the offence and foregrounds their own culpability; this involves strategically sacrificing 'competence face' in order to enhance 'morality face', in a mechanism that I have termed 'face compartmentalization' (cf. Section 4). Some accounts in the corpus lack an explicit evaluative component. These are typically very brief characterizations of the incident (e.g. your experience, the service you received), and they are neither downgraded nor upgraded. Such neutral accounts are not the concern of this study.

Both downgrading and upgrading are performed via a range of lexical resources and syntactic structures; Ogiermann (1999a: 120) notes that downgrading and upgrading effects cannot be simply mapped onto linguistic forms, as they are highly context-sensitive. In the corpus analyzed for this study, the most frequent means of downgrading on the level of discourse semantics are the following:

- Downplaying or blurring responsibility:

- the incident is described as a misunderstanding, confusion or mix-up

- Downplaying the impact (severity) of the incident:

- the incident is described as an inconvenience or an issue

- a frequent strategy in such accounts is to avoid using negatively evaluative lexical items by instead using positively evaluative items which are either directly negated or otherwise presented as an ideal which was not achieved (your visit wasn't a 100\% enjoyable experience; the customer service you received was less than satisfactory on this occasion; you were not entirely happy with your experience; you felt your food was not up to our usual high standard). Choices such as these enable respondents to 'smuggle' into the text lexical items with positive denotations or connotations, while minimizing the occurrence of items with negative denotations or connotations.

- Foregrounding the atypical nature of the incident:

- adverbial phrases describing the incident as an isolated, rare or oneoff occurrence (I'm sorry that you weren't impressed on this particular occasion) 
- Foregrounding the subjective nature of the complainant's experience and the negative evaluation expressed in the review:

- restating the complaint as part of an if-clause, which affects the epistemic modality of the proposition (I'm sorry if you felt in any way that the management were being rude)

- restating the complaint using verbs (e.g. feel, find, perceive, seem, think) that indicate the subjective nature of the complainant's claims (I'm sorry you felt the way we handled your reservation to be unsatisfactory; I'm sorry if you found it unacceptable; my apologies if you perceive it that way; I am sorry if the menu seemed limited to you; I'm sorry to hear that you thought our drinks were too expensive)

Upgrading resources in the corpus fall into three main categories:

- Explicit negative evaluations of S's own performance:

- various lexical resources expressing negative evaluation (the food, service, staff behaviour, etc. are described as appalling, awful, inappropriate, not good enough, not satisfactory, poor, substandard, terrible, unacceptable, unprofessional, wrong, etc.)

- statements to the effect that the incident should not have happened

- Explicit acceptance of S's (the restaurant's) role in or responsibility for the incident:

- in such statements, $\mathrm{S}$ is typically encoded as the agent of the clause (It looks like we failed on more than one occasion on your visit; I am upset that my staff have acted in such a way; we have fallen short of the mark; That is an outrageous mistake on our part; This was a clear miscommunication between staff members, for which we take full responsibility; I take full responsibility for my lack of communication with my staff)

- speakers may foreground their own culpability by explicitly accepting the lack of excuse or mitigating circumstances (I have no excuses, it was just not good enough and we have failed to meet our standards; We hold our hands up and apologise unreservedly for what occurred - no excuses, no mitigating circumstances, on this occasion we got it wrong)

- Explicit acknowledgement of the restaurant's failure to meet its relational obligations to the customer; this adds an extra layer of upgrading by foregrounding the negative impact on $\mathrm{H}$ :

- We let you down; Obviously we have not fulfilled our responsibilities to you on this occasion; I am terribly sorry for you not receiving the customer service you deserved. 
The various means of downgrading and upgrading listed above do not lend themselves to a fine-grained quantification because one utterance may fall into more than one category at the same time. Nevertheless, for the purposes of this study it is sufficient to give quantity data on a relatively general level. Figure 3 shows the total number of utterances in the corpus performing the function of either downgrading or upgrading accounts:

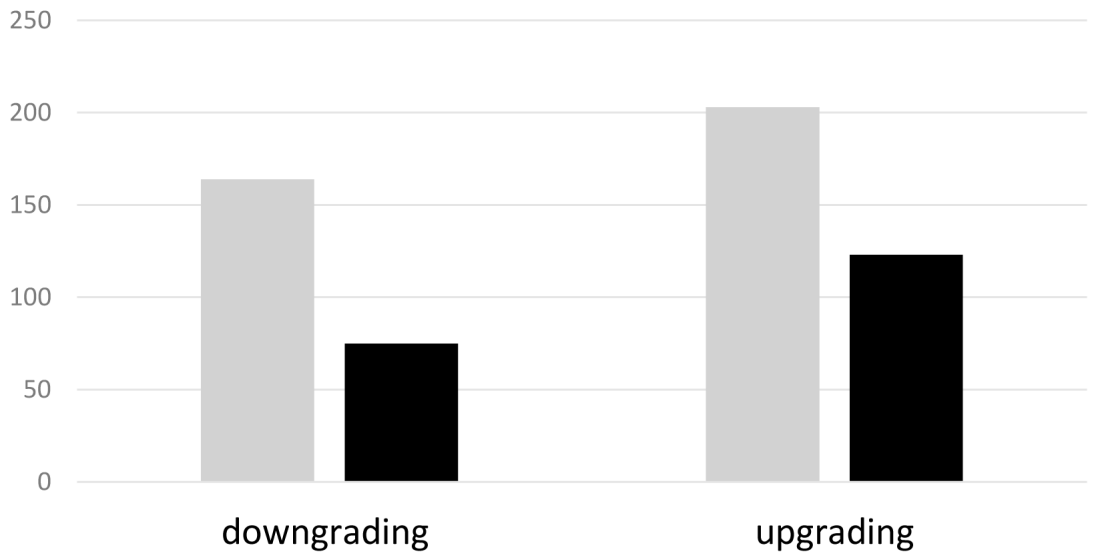

\section{L1 ELF}

Figure 3: Accounts - downgrading and upgrading resources (No. of utterances)

The most obvious observation from the data is that the L1 speakers simply do more of this type of pragmatic work than their ELF counterparts; the L1 responses are more 'pragmatically saturated' in terms of both downgrading and upgrading, while the ELF responses are more neutral in character. The most plausible explanation for this is connected with linguistic competence, as the use of the downgrading/upgrading strategies listed above presupposes a level of proficiency that may be beyond the reach of some ELF speakers. In other words, the pragmatic properties of the texts are to some extent determined by speakers' lexicogrammatical competence. This, like the issue of illocutionary clarity discussed in the previous subsection (5.1), can be viewed from two opposing perspectives. If we measure ELF against the standards of native speaker (L1) language use, then the ELF responses may appear to be defective bare, stripped-down versions of the L1 responses, amounting to a pale shadow of their pragmatically richer L1 counterparts. However, if we view ELF as a fully 
functional variety of English in its own right, operating on its own terms rather than aiming to imitate native speakers' use of language, then we can observe simply that pragmatic (facework) practices in this particular component of the genre are evidently somewhat different in ELF than in L1 English, with the ELF responses tending towards a somewhat more face-neutral tenor. Indeed, ELF users' genre expectations may well be conditioned by these practices, meaning that the prevailing pragmatic norms for ELF responses are not identical to those of $\mathrm{L} 1$ responses.

\section{Conclusion}

The research presented in this article has sought to contribute to the growing body of work exploring the pragmatics of English as a lingua franca (ELF) by offering insights into the similarities and differences between the practices used by L1 and ELF speakers when performing public apologies. The focus was on two key components of the apologies - illocutionary force indicating devices (IFIDs) and accounts of the original incident. The analysis explored both the occurrence (frequency) and linguistic realizations of apologies in order to reveal differing preferences between L1 and ELF speakers with regard to the strategies used in the apologies and their typical linguistic realizations.

Two main points of difference are apparent. Firstly, with regard to IFIDs, the occurrence of sorry with ambiguous illocution is markedly more frequent among the ELF speakers than among their L1 counterparts. Rather than this reflecting a deliberate use of sorry to perform 'non-apologies', I have argued that a more plausible explanation would be that many ELF speakers are in fact constrained by their relatively limited linguistic and pragmatic competence, which sometimes causes them to unwittingly produce utterances with ambiguous illocution. I also argue that this ambiguity should not necessarily be viewed as a case of pragmatic failure or an example of defective language use; rather, sorry may be used by ELF speakers as a pre-packaged, ready-made 'chunk', a device of convenience which can be deployed almost universally to express S's stance whenever writing an apology. Indeed, the primary purpose of this chunk is not to convey any precise desired illocution, but rather to facilitate the writing process.

Secondly, examining the occurrence of facework strategies (downgrading and upgrading) in respondents' accounts of the original incident which led to the complaint, we can observe that the L1 responses are more 'pragmatically saturated' in terms of both downgrading and upgrading, while the ELF responses are more face-neutral in character. Again I argue that this is likely to be caused by many ELF speakers' relatively limited linguistic and pragmatic competence - yet, as before, it should not necessarily be viewed as a defect, but merely as a 
manifestation of somewhat different generic pragmatic norms (expectations) in ELF compared with L1.

Of course, the data analyzed for this study provides only a relatively small snapshot of the genre; its size and scope are restricted due to the labour-intensive nature of the manual analysis, and the tentative conclusions of this particular study may not necessarily be supported by analyses of other samples of L1 and ELF. In the future it would be desirable to enlarge the corpus to include data from other locations, as well as exploring other types of speech acts found in this genre (e.g. requests for the complainant to contact the respondent) in order to test the general validity of the observations presented here. These remain tasks for future studies.

\section{Acknowledgement}

This article is an output of the internal grant project SGS20/FF/2016 (Genres and New Media) at the University of Ostrava.

\section{References}

Austin, J. L. (1975) How To Do Things With Words. $2^{\text {nd }}$ ed. Cambridge, MA: Harvard University Press.

Bella, S. (2014) 'A contrastive study of apologies performed by Greek native speakers and English learners of Greek as a foreign language.' Pragmatics 24/4, 679-713.

Benoit, W. (1995) Accounts, Excuses and Apologies: A Theory of Image Restoration Strategies. New York: State University of New York Press.

Bergman, M. L. and Kasper, G. (1993) 'Perception and performance in native and nonnative apology.' In: Kasper, G. and Blum-Kulka, S. (eds) Interlanguage Pragmatics. Oxford and New York: Oxford University Press. 82-107.

Bhatia, V. K. (1993) Analysing Genre: Language Use in Professional Settings. London: Longman.

Bhatia, V. K. (2004) Worlds of Written Discourse. A Genre-Based View. London and New York: Continuum.

Blum-Kulka, S., House, J. and Kasper, G. (1989) Cross-Cultural Pragmatics: Requests and Apologies. Norwood, NJ: Ablex Publishing Corporation.

Carey, R. (2013) 'On the other side: Formulaic organizing chunks in spoken and written academic ELF.' Journal of English as a Lingua Franca 2/2, 207-228.

Davies, B. L., Merrison, A. J. and Goddard, A. (2007) 'Institutional apologies in UK higher education: Getting back into the black before going into the red.' Journal of Politeness Research 3, 39-63.

Dynel, M. (2014) 'Participation framework underlying YouTube interaction.' Journal of Pragmatics 73, 37-52.

Fetzer, A. (2011) 'Challenges in contrast: A function-to-form approach.' In: Aijmer, K. (ed.) Contrastive Pragmatics. Amsterdam and Philadelphia: John Benjamins. 73-96. 
Gruber, M. C. (2011) 'Pseudo-apologies in the news.' In: Yuasa, E., Bagchi, T. and Beals, K. (eds) Pragmatics and Autolexical Grammar. Amsterdam and Philadelphia: John Benjamins. 93-106.

Harris, S., Grainger, K. and Mullany, L. (2006) 'The pragmatics of political apologies.' Discourse and Society 17/6, 715-737.

Haugh, M. (2013) 'Im/politeness, social practice and the participation order.' Journal of Pragmatics 58, 52-72.

Holmes, J. (1988) 'Paying compliments: A sex-preferential politeness strategy.' Journal of Pragmatics 12, 445-465.

Holmes, J. (1990) ‘Apologies in New Zealand English.' Language in Society 9, 155-199.

House, J. (2013) 'Developing pragmatic competence in English as a lingua franca: Using discourse markers to express (inter)subjectivity and connectivity.' Journal of Pragmatics 59, 57-67.

Jucker, A. H. and Taavitsainen, I. (2008) 'Apologies in the history of English: Routinized and lexicalized expressions of responsibility and regret.' In: Jucker, A. H. and Taavitsainen, I. (eds) Speech Acts in the History of English. Amsterdam and Philadelphia: John Benjamins. 229-244.

Kádár, D. Z. and Haugh, M. (2013) Understanding Politeness. Cambridge: Cambridge University Press.

Kampf, Z. (2009) 'Public (non-)apologies: The discourse of minimizing responsibility.' Journal of Pragmatics 41, 2257-2270.

Kecskes, I. (2013) Intercultural Pragmatics. Oxford: Oxford University Press.

Lee, C. (2016) 'Understanding refusal style and pragmatic competence of teenage Cantonese English learners in refusals: An exploratory study.' Intercultural Pragmatics 13/2, 257-282.

Leech, G. (2014) The Pragmatics of Politeness. Oxford: Oxford University Press.

Lorenzo-Dus, N. (2001) 'Compliment responses among British and Spanish university students: A contrastive study.' Journal of Pragmatics 33, 107-127.

Maíz-Arévalo, C. (2014) 'Expressing disagreement in English as a lingua franca: Whose pragmatic rules?' Intercultural Pragmatics 11/2, 199-224.

Márquez Reiter, R. (2000) Linguistic Politeness in Britain and Uruguay: A Contrastive Study of Requests and Apologies. Amsterdam and Philadelphia: John Benjamins.

Martin, J. R. (1997) 'Analysing genre: Functional parameters.' In: Christie, F. and Martin, J. R. (eds) Genres and Institutions: Social Processes in the Workplace and School. London: Cassell. 3-39.

Martin, J. R. and Rose, D. (2008) Genre Relations: Mapping Culture. London: Equinox.

Mauranen, A. (2009) 'Chunking in ELF: Expressions for managing interaction.' Journal of Intercultural Pragmatics 6/2, 217-233.

Meierkord, C. (1996) Englisch as Medium der interkulturellen Kommunikation. Untersuchungen zum non-native-non-native speaker-Diskurs. Frankfurt am Main: Lang.

Metsä-Ketelä, M. (2016) 'Pragmatic vagueness: Exploring general extenders in English as a lingua franca.' Intercultural Pragmatics 13/3, 325-351.

Ogiermann, E. (2009a) On Apologising in Negative and Positive Politeness Cultures. Amsterdam and Philadelphia: John Benjamins.

Ogiermann, E. (2009b) 'Politeness and in-directness across cultures: A comparison of English, German, Polish and Russian requests.' Journal of Politeness Research 5/2, 189-216. 
Pullin, P. (2013) 'Achieving “comity": The role of linguistic stance in business English as a lingua franca (BELF) meetings.' Journal of English as a Lingua Franca 2/1, 1-23.

Page, R. (2014) 'Saying 'sorry': Corporate apologies posted on Twitter.' Journal of Pragmatics 62, 30-45.

Suszczyńska, M. (1999) 'Apologizing in English, Polish and Hungarian: Different languages, different strategies.' Journal of Pragmatics 31, 1053-1065.

Swales, J. M. (1990) Genre Analysis: English in Academic and Research Settings. Cambridge: Cambridge University Press.

Toury, G. (1995) Descriptive Translation Studies and Beyond. Amsterdam and Philadelphia: John Benjamins.

Trosborg, A. (1995) Interlanguage Pragmatics. Requests, Complaints and Apologies. Berlin: Mouton de Gruyter.

Válková, S. (2012) Regulating Discourse: Compliments and Discourse Signposts (English-Czech Interface). Saarbrücken: Lambert Academic Publishing.

Walkinshaw, I. and Kirkpatrick, A. (2014) 'Mutual face preservation among Asian speakers of English as a Lingua Franca.' Journal of English as a Lingua Franca 3/2, 269-291.

\section{Sources}

Manchester subcorpus (L1):

https:/www.tripadvisor.co.uk/Restaurants-g187069-Manchester_Greater_Manchester_ England.html

Prague subcorpus (ELF):

https://www.tripadvisor.co.uk/Restaurants-g274707-Prague_Bohemia.html

(all retrieved March-August 2016) 


\section{Appendix}

The following tables give the quantitative data that forms the basis for the graphic representations in the main text of the article.

\begin{tabular}{|l|l|l|}
\hline & Manchester & Prague \\
\hline apolog- & 259 & 160 \\
\hline sorry (clear IFID of apology) & 119 & 118 \\
\hline sorry (unclear illocution) & 46 & 137 \\
\hline total & 424 & 415 \\
\hline
\end{tabular}

* apolog- = performative verb apologize or structures incorporating the noun apology/apologies Table 1: IFIDs of apology (total no. of occurrences)

\begin{tabular}{|l|l|l|}
\hline & Manchester & Prague \\
\hline sorry as unambiguous apology IFID & 108 & 112 \\
\hline sorry solely as index of affective stance & 198 & 97 \\
\hline sorry with ambiguous illocution & 43 & 122 \\
\hline total occurrences of sorry & 321 & 310 \\
\hline
\end{tabular}

Table 2: Functions of sorry (all occurrences of sorry in the corpus with a particular function)

\begin{tabular}{|l|l|l|}
\hline & Manchester & Prague \\
\hline upgrading & 203 & 123 \\
\hline downgrading & 164 & 75 \\
\hline
\end{tabular}

Table 3: Accounts - downgrading and upgrading resources (no. of utterances)

Chris Hopkinson is Assistant Professor of English Linguistics at the University of Ostrava, Czech Republic. His research interests range from translation studies to socio-pragmatic discourse analysis, with a particular emphasis on facework, relational work and (im)politeness in online settings. His current research focuses on pragmatic aspects of ELF (English as a Lingua Franca) in emerging genres.

Address: Mgr. Christopher Hopkinson, Ph.D., Department of English and American Studies, Faculty of Arts, University of Ostrava, Reální 5, 70103 Ostrava, Czech Republic. [e-mail: christopher.hopkinson@osu.cz] 